\title{
Synthesis, Ultra-Microscopic and Comparative Studies of Silver and Nickel Nanoparticles
}

\author{
Giriraj Tailor ( $\nabla$ giriraj.tailor66@gmail.com ) \\ Mohanlal Sukhadia University \\ Jyoti Chaudhary \\ M.L.S. University \\ Deepshikha Verma \\ Mewar University
}

\section{Technical Report}

Keywords: Nanoparticles, SEM, TEM, AFM, Nickel and Silver

Posted Date: May 18th, 2020

DOI: https://doi.org/10.21203/rs.3.rs-28745/v1

License: (a) (i) This work is licensed under a Creative Commons Attribution 4.0 International License. Read Full License 


\section{Abstract}

Silver and nickel nanoparticles were synthesized from urea and formaldehyde by using chemical method, followed by calcination at $8000 \mathrm{C}$. The chemical composition and crystallographic structure of silver and nickel nanoparticles were confirmed by XRD. Surface imaging studies like AFM was carried out for estimating surface morphology and particles size distribution. The spherical and porous like morphology of silver and nickel nanoparticles confirmed by SEM and TEM. White spots are observed in the presence of silver and nickel metal ions in spherical forms. The particles size of silver and Nickel metal ions were found to be obtained through XRD 41.43 $\mathrm{nm}$ and $13.71 \mathrm{~nm}$ respectively.

\section{Introduction}

Polymer materials are widely used in industry due to their ease of production, lightweight and ductile nature. A very effective approach to improve mechanical properties is to add fibres, whiskers, platelets or particles as reinforcements to the polymer matrix. The polymer nanoparticles show enhanced properties by the incorporation of low amount of Nano fillers such as carbon black (CB), carbon nanotubes(CNTs), graphene and Nano clay (Huang, 2002, Moniruzzaman et al. 2006, Kim et al. 2010, Fischer, 2003). Polymers have been filled with several inorganic compounds, either synthetic or natural, in order to increase heat and impact resistance, flame retardancy and mechanical strength, and to decrease electrical conductivity and gas permeability with respect to oxygen and water vapor (Hajji et al. 1999). Such composites are widely used in many areas like in electronics, catalysis, transportations and construction, because of their novel properties. Compared to conventional composite nanoparticles, these have ultrafine nanometer size phase dimensions and offer unique combination of properties due to the size(Sanchez et al. 2000, Sanchez et al. 1999, Pomogilo,2000, Novak,1993, Lichtenha et al. 2001, Sanchez et al. 1994, Ells et al. 1999, Kwiatko et al. 2000, Schubert et al. 1995, Mori Kawa et al. 1992, Giannelis et al. 1999, Jordan et al. 2005). Following these approaches, polymers can be improved while keeping their lightweight and ductile nature (Akita et al. 1999, 1999, 1999, Chang et al. 2002, Zavyalov et al. 2002). Another important aspect of nanoscale reinforcements is that it has exceptional potential to generate new phenomena, which give special properties in these materials. Urea- Formaldehyde (UF) resin is widely used as thermosetting materials due to low cost and good thermal and mechanical properties including chemical resistance. This resin is the condensation product of urea and formaldehyde. Most thermosetting resins like UF are used with reinforcing fillers or fibres to produce composite materials for use in a wide range of commodity, engineering and other special applications. Several preparative methods of composite particles of polymer and magnetite particles are proposed ( Ma et al. 2005) like suspension polymerization (Horak et al. 2010) dispersion polymerization (Pimpha et al. 2015) emulsion polymerization (Wang et al. 2005) soap-free emulsion polymerization (Mori et al. 2007) mini emulsion polymerization (Liu et al. 2003) and micro emulsion polymerization (Chaudhary et al. 2017). Above these methods involve for nanoparticles formations require high temperature and expensive instruments. One of the simplest and cost effective techniques to prepare the silver and nickel nanoparticles is by thermal decomposition. This technique has various advantages over other method which include easy handling reaction and short reaction time for the preparation of different type of nanoparticles (Traversa et al. 1998, Farhadi et al. 2010). The objective of this study to synthesized. silver and nickel nanoparticle is performed by using urea and formaldehyde resin as precursor. with thermal decomposition method. The synthesized nanoparticles have been characterized using Scanning Electron Microscopy (SEM), Transmission Electron Microscopy (TEM) and Atomic Force Microscopy (AFM).

\section{Material And Methods}

Silver nitrate $\left(\mathrm{AgNO}_{3}\right)$, Nickel Chloride Hexahydrate $\left(\mathrm{NiCl}_{2} \cdot 6 \mathrm{H}_{2} \mathrm{O}\right)$, Formaldehyde $(\mathrm{HCHO})$ and Urea $\left(\mathrm{NH}_{2} \mathrm{CONH}_{2}\right)$ were purchased from central drug House Pvt. Ltd. and Sodium Hydroxide $(\mathrm{NaOH})$, hydrochloride Acid $(\mathrm{HCl})$ was Purchased from fisher scientific. All solvent was of analytical reagent grade and water used without further purification for the preparation of metal solutions.

Silver and Nickel nanoparticle was synthesized by following methods-

A. Formation of polymer metal complex

B. Formation of silver/ nickel nanoparticle

\section{(A) Formation of Polymer metal complex}

Page 2/12 
1.38 mole of urea, 1.13 moles of formaldehyde and $15 \mathrm{ml}$ glacial acetic acid add in three necked round bottom flask equipped with reflux condenser and stirrer. The mixture was agitated and was allowed cool off. Water was removed by slowly raising the temperature to $60^{\circ} \mathrm{Cand}$ applying a vacuum by means of water pump. This temperature was maintained till the melting of sample which on cooling solidifies into a white colored solid. Then add $15 \mathrm{ml}$ of silver nad nickel metal solution was added to prepare polymer metal complex respectively. The reaction is exothermic in nature. The dried solid sample was purified by the washing with distilled water.

\section{(B) Formation of silver and nickel nanoparticles:}

The polymer metal complex calcinated at $800^{\circ} \mathrm{C}$ for 45 minutes in muffle furnace. Black colored silver and nickel nanoparticles were obtained (Chaudhary et al. 2017, 2018).

\section{Purification of silver and nickel nanoparticles:}

Metallic ions were removed from the nanoparticle by keeping it in $12 \mathrm{~N}$ hydrochloric acid solution for 24 hours. The mixture was centrifuged and washed with distilled water till hydrochloric acid was completely removed (Chaudhary et al. 2018). The volatile impurities got separated at time of calcinations.

\section{Result And Discussion}

Morphological Studies:

SEM images of silver nanoparticles are shown in Figure $1(A)$ and (B). The grey coloured bulk regions indicate bulk polymer matrix and white spherical spots are the distribution of silver nanoparticles. The surface is completely rough due to homogeneous distribution of silver particles which are aggregated on polymer surface.

SEM images of nickel nanoparticles are shown in Figure $2(A)$ and (B). The spherical pores are present on the polymer surface. The morphology of nickel nanoparticles shows the carbon black porous like structure and some particles shows granular type structure.

Figure. 1. SEM images of silver nanoparticles (A) 30000 (B) 20000 Magnification

Figure 2.SEM images of nickel nanoparticles (A) 10000 (B) 30000 Magnification

Figure 3.TEM images of Silver nanoparticles at (A) $100 \mathrm{~nm}$ and (B) $10 \mathrm{~nm}$

Figure 4.TEM images of Nickel nanoparticles at (A) $50 \mathrm{~nm}$ and (B) $20 \mathrm{~nm}$

Interestingly, the nanoparticles of silver were found to be white spherical on rough surface of polymer matrix while nickel nanoparticles were carbon black porous along with some granular ones. Microstructural characterization studies were conducted to 
determine the size of nanoparticles, homogeneity and size distribution. TEM image of silver nanoparticles Figure 3 (A) and (B) shows globular as well as rod like structure on the polymer surface. The black coloured spot on polymer surface indicates metal particles and white bulk part is polymer matrix. The TEM image of nickel nanoparticles Figure $4(A)$ and $(B)$ shows bulk granule like morphology. The black colour present in polymer surface are metal particles and white part is polymer matrix. Thus, SNPs were recorded to be globular and rod like whereas nickel nano particles were of granular morphology.

\section{X-ray Diffraction (XRD) Analysis:}

Table-1 XRD peak list of silver nanoparticles

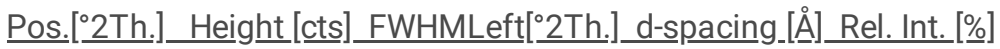

\begin{tabular}{lllll}
32.4336 & 62.16 & 0.2362 & 2.76052 & 2.55 \\
\hline 38.3467 & 2442.12 & 0.2362 & 2.34736 & 100.00 \\
\hline 44.5116 & 976.67 & 0.2657 & 2.03552 & 39.99 \\
\hline 46.4111 & 29.77 & 0.3542 & 1.95654 & 1.22 \\
\hline 64.6085 & 364.99 & 0.2657 & 1.44258 & 14.95 \\
\hline 77.5439 & 290.57 & 0.1771 & 1.23109 & 11.90
\end{tabular}

Figure 5.XRD patterns of silver nanoparticles

Table-2 XRD peak list of nickel nanoparticles

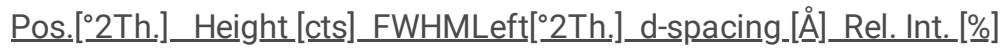

\begin{tabular}{lllll}
43.5115 & 63.77 & 0.4723 & 2.07996 & 100.00 \\
\hline 50.4315 & 26.39 & 0.9446 & 1.80959 & 41.39
\end{tabular}

Figure 6. XRD patterns of nickel nanoparticles

Table :3 Calculations of XRD for Silver Nanoparticles 


\begin{tabular}{lllllllll} 
FWHM & $\begin{array}{l}\text { FWHM in } \\
\text { radian }\end{array}$ & 2Theta & theta & $\begin{array}{l}\text { angle in } \\
\text { radia }\end{array}$ & Cos & d (A) & $\begin{array}{c}\text { Calculated } \\
\text { Size }\end{array}$ \\
\hline 0.1771 & 0.003089411 & 16.49 & 8.245 & 0.143829444 & 0.989674364 & 453.4865157 & & \\
\hline 0.2066 & 0.003604022 & 32.0382 & 16.0191 & 0.2794443 & 0.961208861 & 400.246163 & & \\
\hline 0.4723 & 0.008239011 & 39.5195 & 19.75975 & 0.344697861 & 0.941177592 & 178.8075019 & 414.3494 & $41.43 \mathrm{~nm}$ \\
\hline 0.1181 & 0.002060189 & 45.7766 & 22.8883 & 0.399273678 & 0.921343594 & 730.4722829 & \\
\hline 0.1771 & 0.003089411 & 56.848 & 28.424 & 0.495840889 & 0.87956895 & 510.2544595 & \\
\hline 0.3542 & 0.006178822 & 66.5049 & 33.25245 & 0.580070517 & 0.836424003 & 268.2873624 & & \\
\hline 0.4723 & 0.008239011 & 75.5318 & 37.7659 & 0.658805144 & 0.790724253 & 212.8297106 & &
\end{tabular}

Table:4 Calculations of XRD for Nickel Nanoparticles

\begin{tabular}{lllllllll} 
FWHM & $\begin{array}{l}\text { FWHM in } \\
\text { radian }\end{array}$ & 2Theta & theta & $\begin{array}{l}\text { angle in } \\
\text { radia }\end{array}$ & Cos & d (A) & \multicolumn{2}{c}{$\begin{array}{c}\text { Calculated } \\
\text { size }\end{array}$} \\
\hline 0.4723 & 0.008239011 & 43.5115 & 21.75575 & 0.379516972 & 0.928843692 & 181.1818452 & 137.0898 & $13.71 \mathrm{~nm}$ \\
\hline 0.9446 & 0.016478022 & 50.4315 & 25.21575 & 0.43987475 & 0.904805005 & 92.99772498 & &
\end{tabular}

The calculated size of the synthesized silver and nickel nanoparticles are obtained $41.43 \mathrm{~nm}$ and $13.71 \mathrm{~nm}$ respectively which corroborates efficacy of the synthetic method utilized.

Atomic Force Microscopy (AFM) Analysis:

Figure 7. AFM image (A) 3D topography and (B) 2D topography of silver nanoparticles

Figure 8. AFM image (A) 3D topography and (B) 2D topography of nickel nanoparticles

Surface imaging studies were performed using AFM for estimating surface morphology and particles size distribution. Spherical shaped metal ions were identified at the surface of silver nanoparticles. The white spots as shown in figure 7 (A) (B) and 8 (A) (B) represent the presence of silver and nickel metal ions respectively in spherical forms. The maximum peak height obtained is of 37.7 $\mathrm{nm}$ and $92.1 \mathrm{~nm}$ for silver and nickel nanoparticles respectively.

The present study supports the findings of (Kotakadi et al. 2013) who have also made the similar observations that AgNPs have a spherical shape and the diameters of AgNPs varied between 27 and $50 \mathrm{~nm}$. Similarly, (Muzamil et al. 2014) have also recorded that particles are nearly spherical identical in shape, dispersive \& crystalline in structure

\section{Conclusion:}

Silver and nickel nanoparticles synthesized by chemical precipitation method followed by thermal decomposition. The morphology studies by SEM show that the silver nanoparticles are spherical and nickel nanoparticles have pore-like shapes like that of carbonblack. The particle size determined by XRD and surface to volume area results of AFM studies also indicate the exact structure and 
probable properties of the synthesized nanoparticles. Our results are good indicative of the efficacy of the chemical precipitation method in the synthesis of the nanoparticles. In future, these Silver Nano Particles can be further analyzed for its heat transfer properties and application in nanofluids and conductive gels. We will further investigate the catalytic activity of nickel nanoparticles.

\section{Abbreviations}

SNPs: Silver Nanoparticles; SEM: Scanning Electron Microscopy; TEM: Transmission Electron Microscopy; AFM: Atomic Force Microscopy; NPs: Nanoparticles; XRD: X-Ray Diffraction; 3D: Three Dimensional; 2D: Two Dimensional; CB: Carbon Black; CNT: Carbon Nano Tube; UF: Urea-Formaldehyde;

\section{Declarations}

\section{Availability of data and materials}

Not applicable. "Please contact with author for data request"

\section{Competing interest}

The authors declare no conflict of interest.

\section{Funding statement}

This research did not receive any specific grant from funding agencies in the public, commercial, or not-for-profit sectors.

\section{Author contributions}

Giriraj Tailor: Conceived and designed the experiments; Analyzed and interpreted the data; Wrote the paper.

Jyoti Chaudhary: Contributed reagents, materials, analysis tools or data.

Deepshikha Verma: Performed the experiments.

\section{Conflicts of Interest:}

The author(s) declare(s) that there is no conflict of interest regarding the publication of this paper.

\section{Acknowledgement:}

The authors are thankful to MNIT Jaipur, Rajasthan, India for providing the characterization facility. 
Akita H and Kobayashi H. (1999). J. Eur. Ceram. Soc 3 p 7209.

Akita H, Hattori T. (1999). Journal of Polymer Science: Part B: Polymer Physics. 37, p 189.

Akita H, Kobayashi H, Hattori T and Kagawa K (1999). J. Eur. Ceram. Soc 37 p 199.

Chang J H and An Y U (2002). J. Eur. Ceram. Soc 40 p 670.

Chaudhary J, Tailor G, Joshi A. and Kumar D Asian J Chem 7 (2017) p 1492.

Chaudhary J, Tailor G, kumar D (2018). Res. J. Chem. Environ. 23,3.

Chaudhary J, Tailor G, Kumar D. Joshi A (2017). Asian J. Chem. 29,7.

Ells Worth M W and D L C (1999). Polymer News 24, p331.

Farhadi S, Rashidi N (2010). Polyhedron, 29, 2959-2965. http://dx.doi.org/10.1016/j.poly.2010.08.019.

Fischer H (2003). Materials Science and Engineering: C. 23, p763.

Gao F (2004). Materials Today 11, 50.

Giannelis E P, Krishnamoorti R and Manios E (1999). Adv Poly Sci 138,108.

Hajji P L, David JF, Gerald JP Pascault and Vigier GJ (1999). Journal of Polymer Science: Part B: Polymer Physics 37 p3172.

Horak D, Trchov M, BeneM. J, Veverka M. and. Pollert E Polymer 5 (2010) p 13116.

Huang J C (2002). Adv Polym Tech 4 p 299.

Jordan J, Jacob K I, Tannenbaum R, Sharaf MA, Jasiuk I. (2005). Materials Science and Engineering: A. p.393111.

Kim H, Abdala A A and Macosko C W. Graphene/polymernanoparticles, (2010). Macromolecules 43 p 6515.

Kotakadi V S, Rao Y S, Gaddam S A, Prasad T N V.K V, Reddy A V, Gopal D S. (2013). Colloids and Surfaces B: Biointerfaces 105, pp 194-198.

Kwiatko W K C and Lukehart C M (2000). Handbook of Nanostructured Materials H S Nalwa academic press CA San Deigo. Lichtenha J D, SchwabJ J and Reinerth W A (2001). J Chem Innovation 31, p 3.

Liu Z L, Ding Z H, Yao K L,Tao J, Du GH, Lu Q, Wang H X Gong F L and X. Chen(2003). J. Magn. Magn. Mater. 26 , p 598. Ma Z Y, Guan Y P, Liu X Q and Liu. H Z Langmuir (2005) p 16987.

Moniruzzaman M and Winey K I. (2006). Macromolecules 16 p5194.

Mori Kawa A, lyoku Y, Kakimoto M and Imai Y (1992). J Mater Chem 2, p 679.

Mori Y. and Kawaguchi H. (2007). Colloids Surf. B p 246.

Muzamil M, Khalid N, Aziz M D and Abbas S A. (2014). IOP Conf. Series: Materials Science and Engineering 60 p 012034.

Novak B M (1993). Adv Mater 5, p 422.

Pimpha, N, Chaleawlert-umpon S and Sunintaboon P Polymer 53 (2012) p 2015.

Pomogilo A D (2000). Russ Chem Rev 69, p 53.

Sanchez C and Ribot F (1994). New J Chem 18, p1007.

Page $7 / 12$ 
Sanchez C, Beau B Le and Ribot F (2000) Sol-Gel Sci Tech 19, p 313.

Sanchez C, F Ribot and B Lebau (1999) J Mater Chem 9, p35.

Schubert U, Husing N and Lorenz A (1995). Chem Mater 5, p2010.

Traversa E, Sakamoto M, Sadaoka Y (1998). Part. Sci. Technol. 16, 185-214. http://dx.doi.org/10.1080/02726359808906794 Wang P C, LeeC F, Young T H, Lin D T and Chiu W Y (2005). J. Polym. Sci. Part. A Polym. Chem. 43, p 1342.

Zavyalov SA, Pivkina A N and Schoonman (2002). J. Solid State lonics 14 p 7415.

\section{Figures}

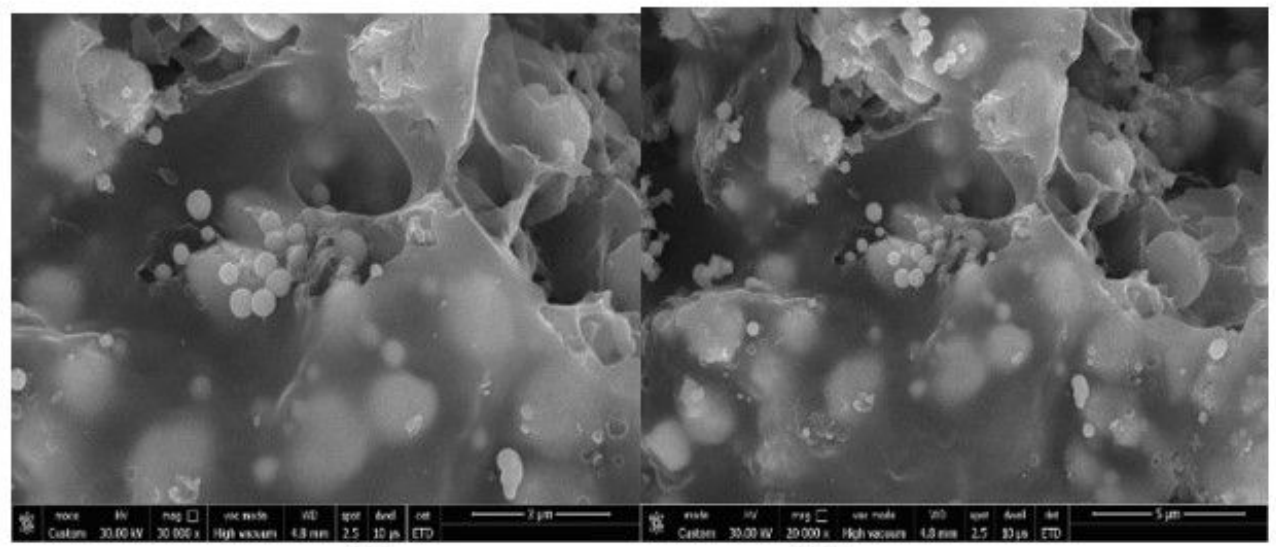

(A)

(B)

\section{Figure 1}

SEM images of silver nanoparticles (A) 30000 (B) 20000 Magnification

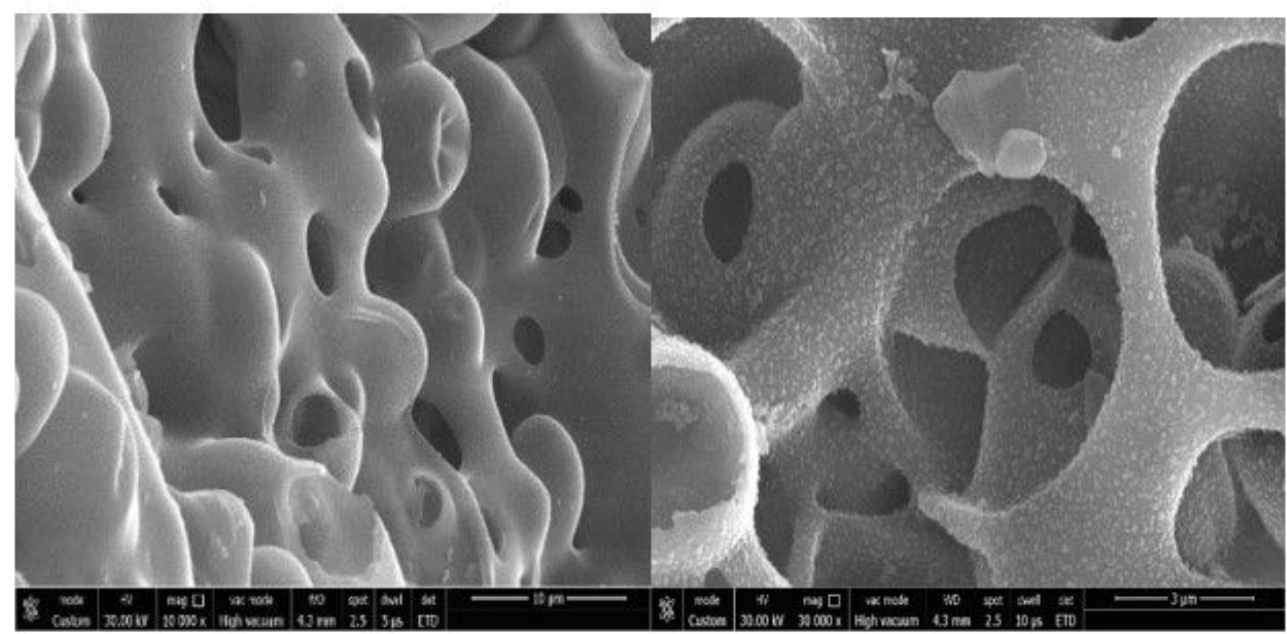

(A)

(B)

\section{Figure 2}

SEM images of nickel nanoparticles (A) 10000 (B) 30000 Magnification 


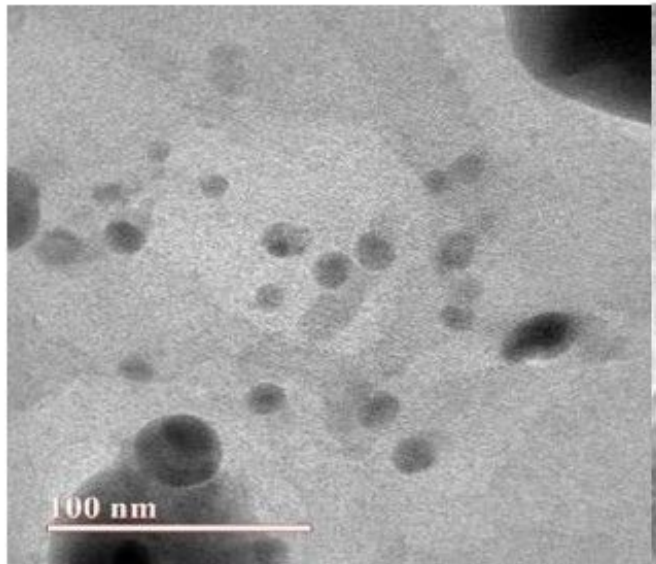

(A)

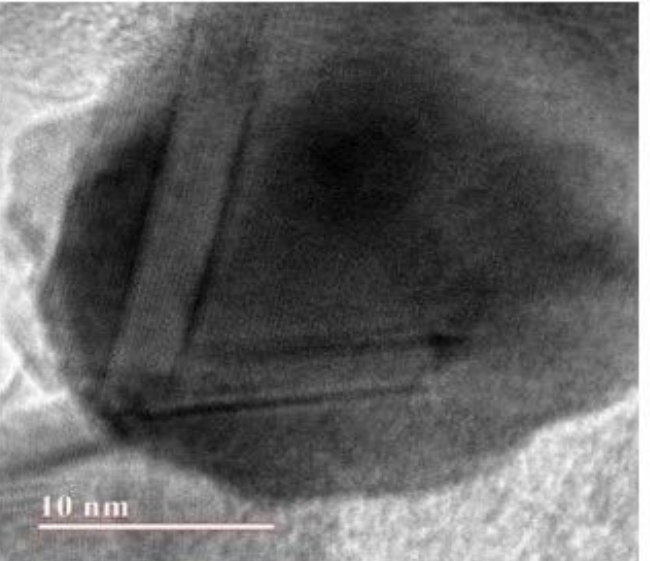

(B)

\section{Figure 3}

TEM images of Silver nanoparticles at (A) 100nm and (B) $10 \mathrm{~nm}$

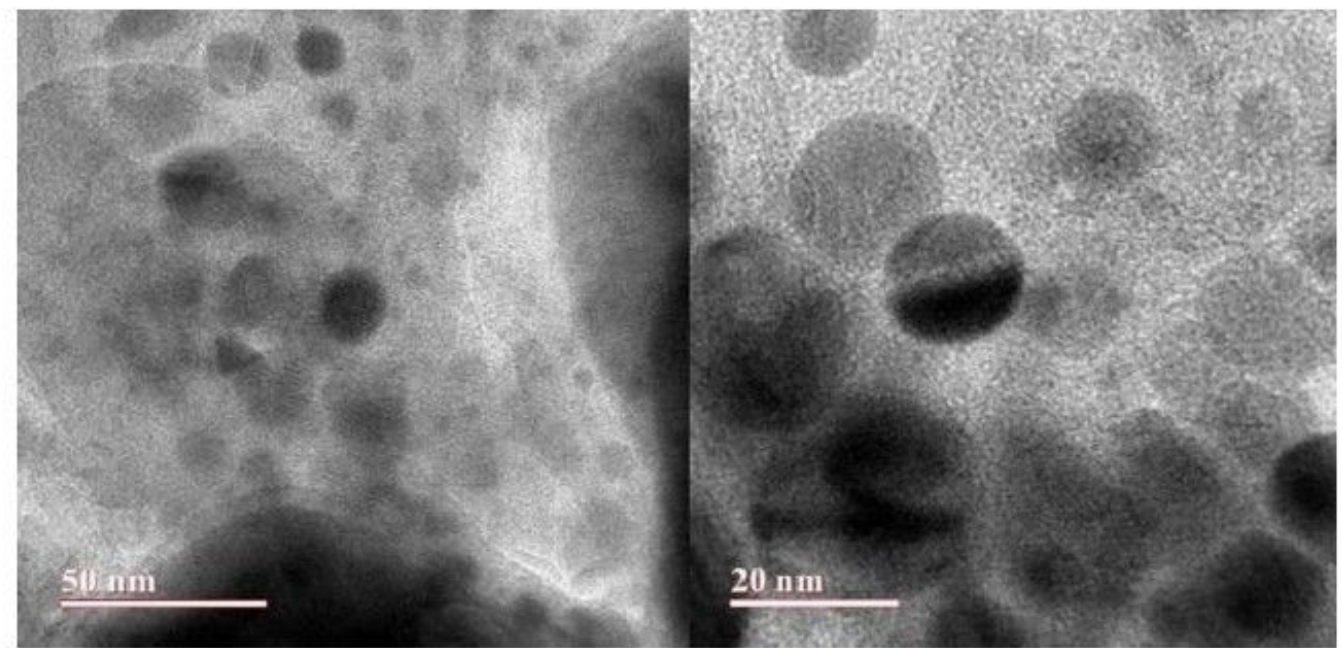

(A)

(Bi)

\section{Figure 4}

TEM images of Nickel nanoparticles at (A) 50nm and (B) $20 \mathrm{~nm}$ 


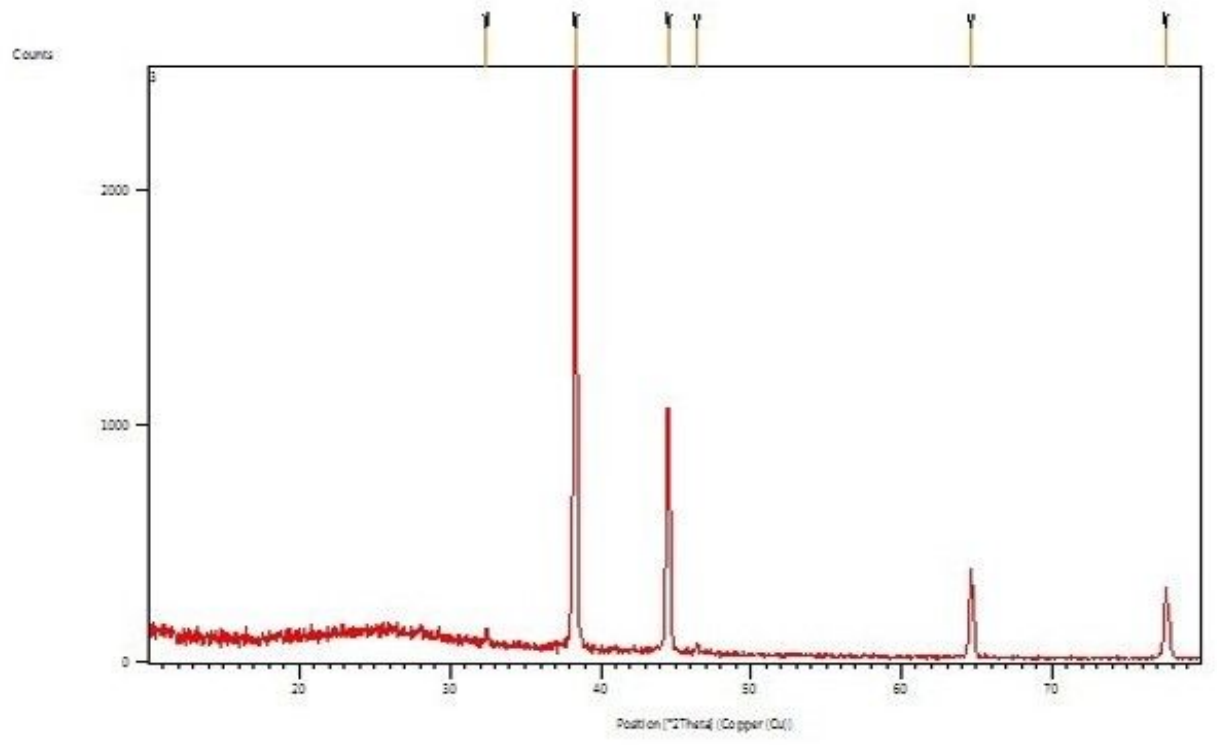

Figure 5

XRD patterns of silver nanoparticles

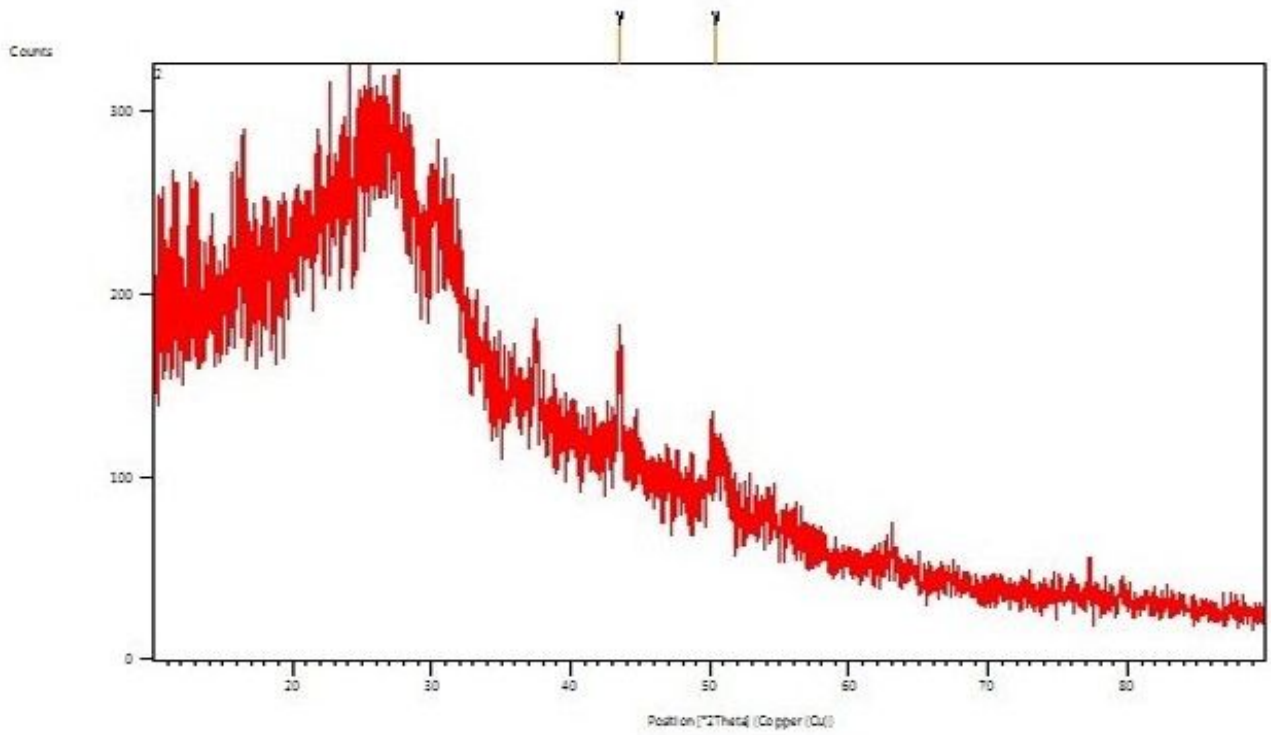

Figure 6

XRD patterns of nickel nanoparticles 


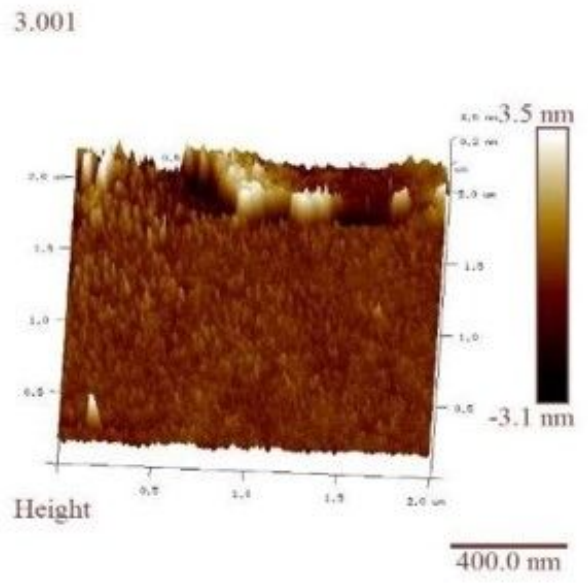

(A)

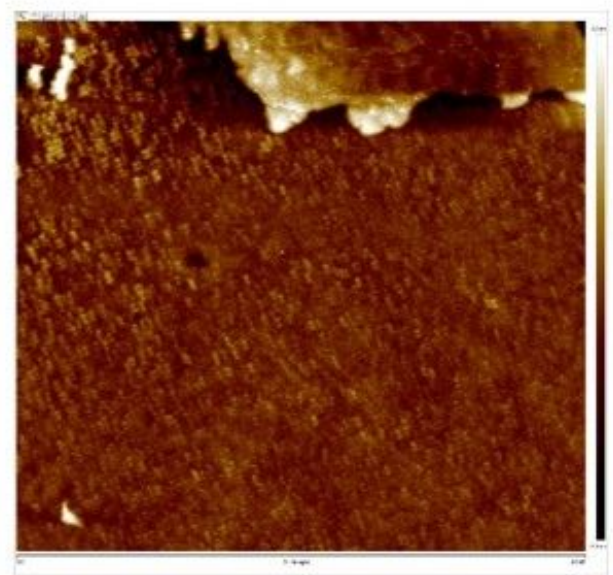

(B)

Figure 7

AFM image (A) 3D topography and (B) 2D topography of silver nanoparticles

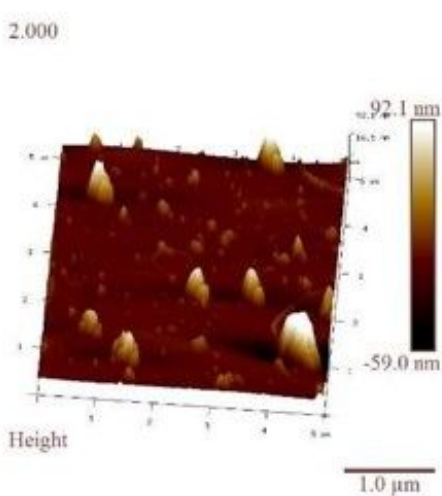

(A)

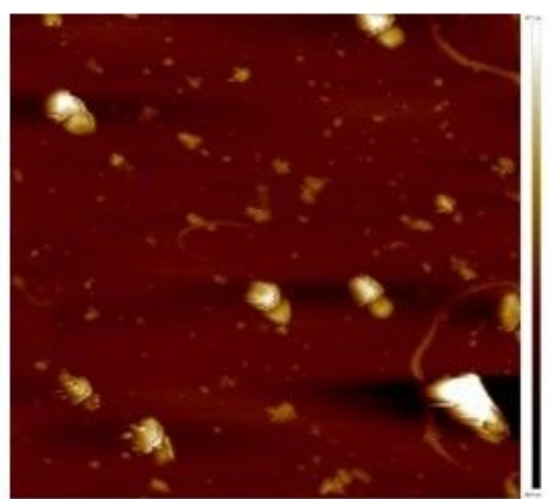

(B)

\section{Figure 8}

AFM image (A) 3D topography and (B) 2D topography of nickel nanoparticles 

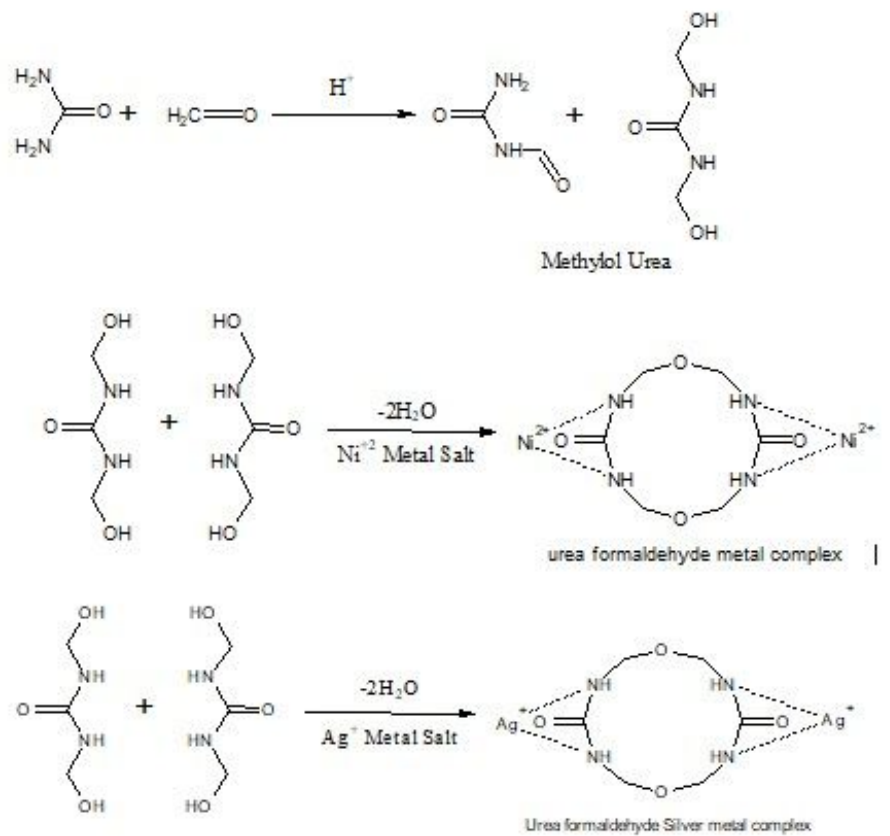

\section{Figure 9}

Scheme 1. Reaction for the nickel and silver nanoparticle 